\title{
Hidrocéfalo ótico secundario a trombosis del seno lateral en niños: Reporte de un caso clínico y revisión de la literatura
}

\author{
Otitic hydrocephalus secondary to lateral sinus thrombosis in children: \\ A case report and literature review
}

\author{
Soto Riquelme I', Sáez Cáceres E², Ibacache Norambuena F², Papuzinski Aguayo C².
}

\begin{abstract}
RESUMEN
La trombosis del seno lateral es una complicación potencialmente mortal de la otitis media aguda, aunque infrecuente en la actualidad debido al amplio uso de antibióticos. Sus manifestaciones clínicas son habitualmente fiebre, cefalea y otalgia, agregándose vómitos, edema de papila y compromiso del estado general. El examen diagnóstico de elección es la resonancia magnética en fase venosa y el manejo incluye terapia antibiótica endovenosa, miringotomía con instalación de tubo de ventilación y mastoidectomía, asociado o no a anticoagulación. Se reporta el caso de un escolar de 9 años derivado al Hospital Carlos Van Buren con un hidrocéfalo ótico secundario a una trombosis del seno lateral, manejado con terapia antibiótica endovenosa, mastoidectomía simple, miringotomía con instalación de tubos de ventilación y anticoagulación, evolucionando favorablemente con regresión de la sintomatología y recanalización del seno en el control imagenológico. Se realiza una revisión bibliográfica sobre la presentación clínica, diagnóstico y manejo de la trombosis del seno lateral en niños como complicación de la otitis media aguda.

Palabras clave: Hidrocéfalo ótico, trombosis del seno lateral, complicaciones de la otitis media aguda.
\end{abstract}

\begin{abstract}
Lateral sinus thrombosis (LST) is a potentially life threatening complication of acute otitis media. However, report rates have been decreasing since the spreaded use of antibiotic prescription. Patient developing LST usually complain about fever, headache and ear pain. Consequently, they may develop vomiting, papilledema and unhealthyness. Magnetic resonance venography (MRV) is considered the gold standard for LST diagnosis. Management procedures include parenteral antibiotics, myringotomy with tympanostomy tube and mastoidectomy, associated or not with anticoagulant therapy. The present study reports the case of a 9-year-old male referred to the Hospital Carlos Van Buren diagnosed with an otitic hydrocephalus secondary to lateral sinus thrombosis,
\end{abstract}

1 Interno de Medicina, Universidad de Valparaíso.

2 Médicos Servicio ORL, Hospital Carlos Van Buren, Universidad de Valparaíso. 
managed with intravenous antibiotic therapy, simple mastoidectomy, myringotomy with tympanostomy tube placement and anticoagulation. Favorable clinical and imagenologic outcomes were obtained. We provide a brief summary about clinical features, diagnosis and management of the LST in children as a complication of acute otitis media.

Key words: Otitic hydrocephalus, lateral sinus thrombosis, acute otitis media complications.

\section{INTRODUCCIÓN}

Las complicaciones intracraneales secundarias de una otitis media aguda (OMA), se presentan en $0 \%$ $7 \%$ de los casos ${ }^{1}$. La trombosis del seno lateral (TSL) es una complicación rara ${ }^{2,3}$, pero potencialmente letal cuando existe una otomastoiditis ${ }^{4}$ secundaria a una OMA.

La posición del seno sigmoideo en el hueso temporal, Io hace particularmente vulnerable a cambios inflamatorios resultantes de la extensión de infecciones del oído medio. La trombosis 0 tromboflebitis puede producirse por extensión directa desde el oído medio y mastoides por coalescencia, 0 por vía hematógena, encontrándose en esos casos el hueso intacto en la intervención quirúrgica ${ }^{5}$. Aparentemente, las bacterias con actividad protrombótica juegan un importante rol en la etiología, por lo que no se recomienda el estudio rutinario de factores protrombóticos a menos que exista el antecedente en la historia del paciente, dado que no tendrían una importancia mayor en el desarrollo de TSL otogénicas ${ }^{6}$. El agente causal se logra aislar en sólo el $46 \%$ de cultivos, siendo los más frecuentemente aislados Streptococcus betahemolítico, Streptococcus pneumoniae y Staphylococcus aureus ${ }^{7}$. A medida que la condición progresa, el trombo se puede propagar a otros senos, inclusive a la vena yugular interna. La oclusión de los vasos finalmente puede interferir en la circulación del líquido cefalorraquídeo (LCR), llevando a elevaciones en la presión intracraneana (PIC) sin signos de hidrocefalia ni evidencia de dilatación ventricular, produciendo lo que se conoce como hidrocéfalo ótico ${ }^{8,9}$.

Los síntomas y signos clínicos de TSL incluyen cefalea, fiebre e infección prolongada del oído, manifestaciones que son compartidas con cuadros de otitis, mastoiditis y otras complicaciones intracraneales. Clásicamente, las manifestaciones clínicas incluyen la fiebre en agujas, signos de sepsis, otalgia intensa, cefalea y aumento de volumen con eritema retroauricular, secundario a una OM no tratada ${ }^{10}$. Sin embargo, la clásica fiebre en patrón de agujas, reflejo de bacteriemia, es de baja frecuencia en la actualidad 5 .

De $26 \%$ a $33 \%$ de los pacientes pediátricos con diagnóstico de TSL de origen otogénico se presenta como un hidrocéfalo ótico $0^{5,11}$. Otras complicaciones de la TSL ocurren en el 10\% de los pacientes e incluyen compromiso de pares craneales, hipoacusia sensorio neural, accidente cerebro vascular y artritis séptica? ${ }^{7}$. Las manifestaciones del hidrocéfalo ótico son comunes a cualquier causa de aumento de la PIC, incluyendo cefalea severa, vómitos, edema de papila y parálisis del VI par con diplopía. Las presiones de apertura en la punción lumbar también se pueden encontrar elevadas 9 .

Es fundamental considerar que la presentación clínica descrita se ve frecuentemente afectada por la utilización de terapia antibiótica previa, haciendo que el diagnóstico de la TSL sea más difícil y se altere el curso clínico esperado ${ }^{12}$.

La confirmación diagnóstica es imagenológica. El signo del delta vacío causado por el engrosamiento de la pared del seno lateral es considerado diagnóstico ${ }^{13}$. Este hallazgo se demuestra con mayor sensibilidad en RM en fase venosa, que corresponde al examen de elección. La RM también es superior para demostrar la formación de abscesos dentro del seno y excluir otras complicaciones como empiema subdural 0 abscesos cerebrales. A pesar de sus múltiples ventajas, es muy costosa y no disponible en muchos centros ${ }^{5}$.

El manejo actual de la TSL consiste en terapia con antibióticos endovenosos e intervención quirúrgica con o sin anticoagulación. La cirugía tradicional incluye la mastoidectomía con o sin miringotomía e instalación de tubos de ventilación, y en algunos casos la ligadura de la vena yugular y extracción del trombo mediante flebotomía y trombectomía ${ }^{8}$. El tratamiento del hidrocéfalo ótico está orientado 
a resolver la causa de base, se ha reportado buena respuesta al tratamiento sintomático con corticoides, acetazolamida y furosemida, respectivamente. Otras terapias como punciones lumbares seriadas, descompresión subtemporal o suboccipital han sido reportadas como inefectivas 9 .

Respecto del pronóstico, en algunos casos el trombo puede embolizar hacia localizaciones distales, aumentando la morbilidad y mortalidad. En la era preantibiótica la mortalidad estimada de esta condición era de $100 \%$ versus $20 \%$ en aquellos en que se realizaba intervención quirúrgica ${ }^{10}$. Cuando se implementó la terapia antibiótica, asociada en muchos casos a anticoagulación, el pronóstico en los pacientes con TSL mejoró significativamente. Actualmente la mortalidad es menor a $1 \%{ }^{7,8}$.

\section{CASO CLÍNICO}

Paciente de 9 años de edad, de sexo masculino, sin antecedentes mórbidos de relevancia. Con cefalea y otalgia derecha, asociado a vómitos explosivos de 5 días de evolución. Consultó en atención primaria, donde se diagnosticó OMA, iniciándose amoxicilina y antiinflamatorios. A pesar del tratamiento evolucionó con fotofobia, diplopía, inestabilidad de la marcha, paresia del VI par izquierdo, VII par derecho y edema de papila, por lo que se decidió hospitalizar en el
Hospital Provincial del Huasco, Vallenar, donde se realizó tomografía computarizada (TC) de encéfalo con contraste que fue informada sin hallazgos patológicos, del resto de los exámenes destacaba PCR de $55 \mathrm{mg} / \mathrm{dL}$ y punción lumbar con aumento subjetivo de la presión de salida de LCR.

Se deriva al Hospital Carlos Van Buren para estudio y manejo de síndrome de hipertensión endocraneana. Al ingreso se encontraba estable, afebril, con cefalea y diplopía. A la exploración física se constató paresia del VI par izquierdo, leve asimetría facial derecha y edema de papila bilateral, coherente con los hallazgos previos. Entre los exámenes realizados destacaba una punción lumbar con presión de apertura de $48 \mathrm{~cm}$ de $\mathrm{H} 2 \mathrm{O}$, diagnosticándose pseudotumor cerebri e indicándose acetazolamida $250 \mathrm{mg}$ cada 8 horas. Se realizó RM en fase venosa que evidenciaba trombosis del seno transverso y sigmoideo derecho con otomastoiditis del mismo lado (Figura 1). Tras la evaluación por neurología infantil se decidió monitorización y manejo en UCI pediátrica. Fue evaluado por neurocirugía, infectología y neurología infantil, agregándose al tratamiento terapia antibiótica endovenosa biasociada con cefotaxima y metronidazol para el cuadro infeccioso, heparina de bajo peso molecular para el manejo de la trombosis y prednisona por 5 días para el manejo del hidrocéfalo ótico.

Tras 36 horas de monitorización, debido a que el paciente se encontraba estable se decidió

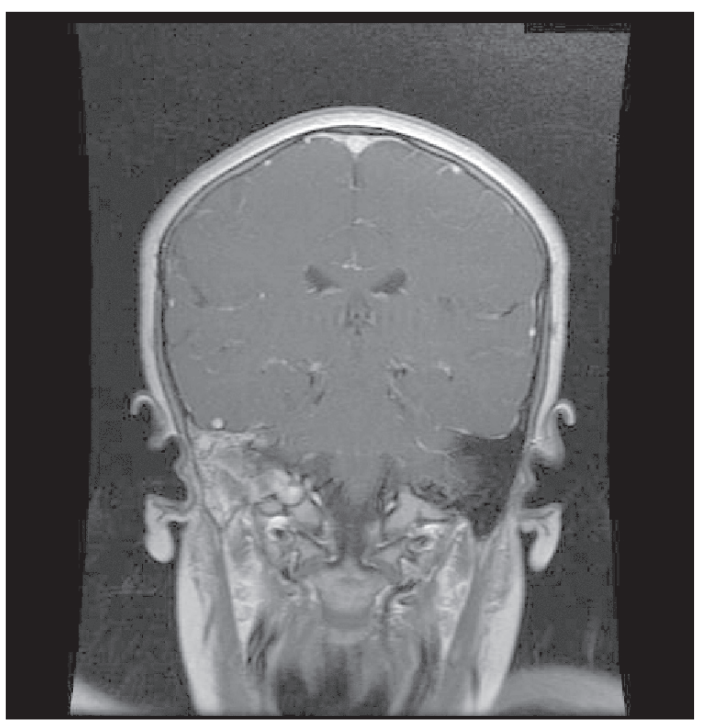

Figura 1. Trombo en seno sigmoides y otomastoiditis. 
traslado al Servicio de Neurología Infantil, donde se realizó TC de oídos que evidenciaba ocupación de la caja timpánica, del ápex petroso y velamiento mastoideo (Figura 2). Fue evaluado por equipo de otorrinolaringología, observándose abombamiento de la membrana timpánica y opacidad a la otoscopía del oído afectado. Debido al compromiso de las celdillas mastoídeas y el hidrocéfalo ótico secundario a la trombosis del seno lateral se indicó resolución quirúrgica, la que fue realizada mediante mastoidectomía simple, miringotomía e instalación de tubo de ventilación. Entre los hallazgos intraoperatorios destacaba la presencia de secreción serohemática en la punción transtimpánica y abundante mucosa inflamatoria en el vértice mastoideo. Se tomaron muestras para cultivo que fueron negativas.

El paciente evolucionó favorablemente, afebril, con escasa otorrea, regresión de la cefalea, corrección del edema papilar y disminución progresiva del compromiso del VI y VII par. Hubo normalización de los índices de actividad inflamatoria. Se indicó ciprofloxacino asociado a corticoides en gotas óticas y se completaron 21 días de tratamiento antibiótico con amoxicilina y ac. clavulánico para el cuadro infeccioso. Para el manejo de la trombosis se decidió cambio a anticoagulación oral con acenocumarol y controles seriados con INR hasta rangos terapéuticos. En la RM en fase venosa de control a los 15 días se observaba recanalización del seno lateral (Figura 3).

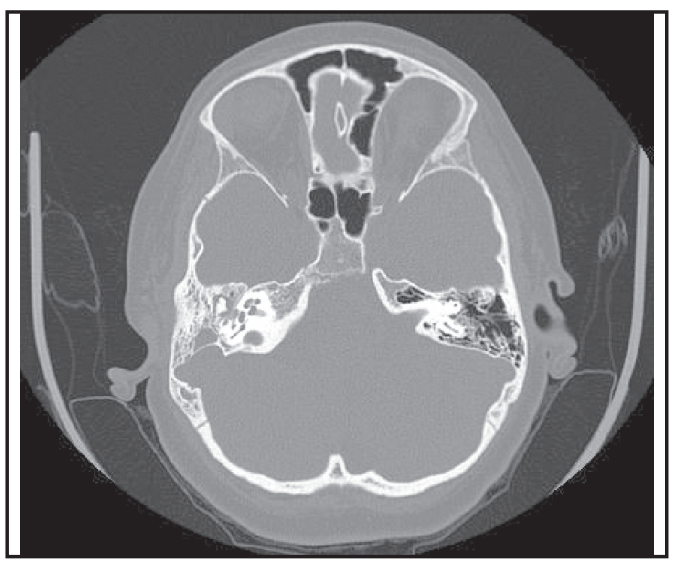

Figura 2. TC de oídos. Otomastoiditis. Se observa ocupación de la caja timpánica y del ápex petroso que se extiende como un velamiento mastoideo.

\section{DISCUSIÓN}

La trombosis del seno lateral secundaria a OM es de baja frecuencia actualmente, debido a la amplia distribución del uso de antibióticos ${ }^{14}$, pero además de la disminución en su incidencia, morbilidad y mortalidad asociada, se ha visto una variación en su presentación y curso clínico.

En los últimos años se ha reportado una predominancia de síntomas de la esfera neurológica sobre la otológica. En una serie de casos de los últimos 10 años en un hospital terciario de EE.UU. el síntoma más frecuente fue la cefalea, casi no hubo fiebre y cuando existió fue de bajo grado, y en casi todos los casos hubo compromiso del VI o VII par asociado a manifestaciones de elevación de la PIC incluyendo cefalea, náuseas, vómitos y fotofobia ${ }^{10}$. Otra revisión de casos ha reportado que el signo más frecuente fue la fiebre en el $88 \%$ de los casos, con hallazgos

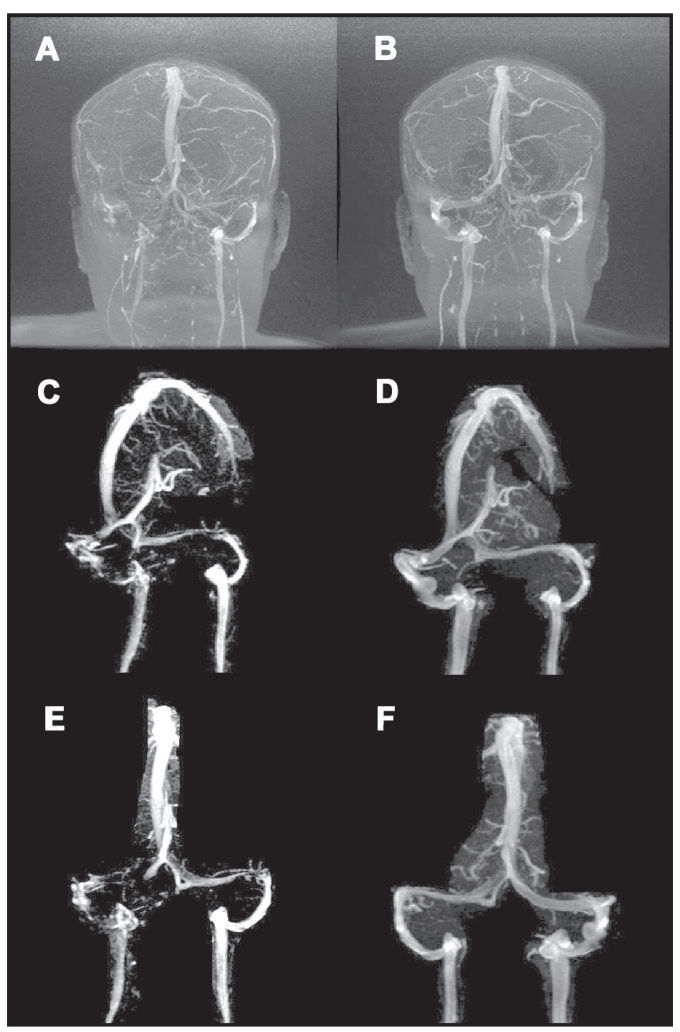

Figura 3. RM en fase venosa. Se observa la trombosis de seno lateral y transverso por RM en fase venosa $(A, C, E)$ y la recanalización en el control imagenológico posterior (B, D, F). 
neurológicos en el $55 \%{ }^{6}$. Cabe destacar que en el primer estudio se describe que la mayoría de los casos fueron tratados previamente con antibióticos, que fue realizado en un centro de alto nivel y que el perfil socioeconómico dista de la realidad descrita en revisiones más amplias que incluyen casos reportados en países con un acceso a la salud limitado.

Respecto de la epidemiología, en una revisión sistemática de casos publicados en los últimos 20 años ${ }^{7}$, se encontró que $73 \%$ de los pacientes con TSL como complicación de OM eran hombres, con una edad promedio de 7,7 años.

Con la ayuda de las técnicas radiológicas modernas que detectan el trombo con alta precisión y sensibilidad, no debería haber dificultades para establecer el correcto diagnóstico ni para un seguimiento adecuado de su posible propagación $n^{5}$. Sobre el examen diagnóstico más adecuado, se ha establecido una sensibilidad del $100 \%$ versus $87 \%$ entre la RM en fase venosa y la TC contrastada?. Por lo que si bien la RM en fase venosa es el examen de elección, frente a sospecha de TSL puede realizarse una TC de encéfalo contrastada, y efectuar una RM en fase venosa si persiste la sospecha frente a una TC sin hallazgos patológicos, como ocurrió en el caso presentado.

En la actualidad, la aproximación terapéutica quirúrgica y médica más apropiada no está claramente establecida. Se describe que el manejo de la infección se realiza con antibióticos de amplio espectro en cerca del $100 \%$ de los casos, utilizándose más frecuentemente una cefalosporina de tercera generación asociada a metronidazol. En cuanto a la práctica quirúrgica, se ha encontrado que en el $94 \%$ de los casos es mastoidectomizado y que en el $50 \%$ existe manipulación del seno trombosado ${ }^{7}$.

Mientras la mayoría de los cirujanos realizan una mastoidectomía simple, con o sin miringotomía, instalación de tubos de ventilación y aspiración del seno si es que existe enfermedad aguda; el tratamiento del trombo, luego de confirmar la ausencia de flujo sanguíneo mediante aspiración, varía desde no más intervención a venotomía y trombectomía. Sin embargo, la verdadera controversia se relaciona con el rol de la anticoagulación de rutina en el manejo de la TSL, lo cual se ve reflejado en que su utilización varía entre 38\%-55\% de los casos ${ }^{6-8}$.
Se ha sugerido precaución frente al uso de rutina de anticoagulantes debido principalmente a las complicaciones hemorrágicas, la evidencia de recanalización sin haber utilizado anticoagulación y recuperación clínica pese a la persistencia del trombo. Por otro lado el sustrato para la utilización de anticoagulación de rutina se fundamenta en su relativa seguridad, en su eficacia para la prevención de embolización o persistencia de trombosis séptica, reducción de secuelas neurológicas a largo plazo y en la evidencia de recanalización en el seguimiento imagenológico cuando se utilizan ${ }^{6,8}$.

Al comparar mediante imágenes en el seguimiento de pacientes con TSL se ha demostrado que existen mayores tasas de recanalización total o parcial de los senos comprometidos en aquellos que fueron anticoagulados ${ }^{7,11}$.

El panorama es distinto para la recuperación clínica, observándose que al parecer no hay evidencia que sugiera que la presencia de recanalización en los controles con imágenes afecte el pronóstico ${ }^{15}$, existe otro caso en Chile de TSL en un preescolar de 5 años reportado en 2012 que fue manejado con anticoagulación, evolucionando con regresión total de la sintomatología pese a que la recanalización del seno lateral fue parcial en los controles imagenológicos a 6 meses $^{12}$.

\section{CONCLUSIÓN}

La trombosis del seno lateral constituye una complicación rara, pero tratable, de la otitis media en la población pediátrica. Los signos y síntomas de compromiso intracraneal asociados al antecedente de otitis media aguda constituyen los elementos de sospecha para el médico, aun si no existe un compromiso del estado general severo o fiebre, debido a que la amplia utilización de antibióticos puede en definitiva enmascarar la sintomatología. La imagenología constituye el elemento diagnóstico principal, y el manejo debe ser con terapia antibiótica endovenosa, cirugía mediante mastoidectomía con la remoción del tejido granulatorio perisinusal. La miringotomía e inserción de tubos de ventilación debe ser considerada principalmente para la enfermedad aguda del oído medio.

Respecto de la recomendación de anticoagulación, dada la dificultad en establecer su utilidad 
en cuanto a morbilidad de TSL, se considera que aún falta evidencia suficiente para responder con certeza su rol en el manejo de la TSL. Pero dada la posibilidad de reducción de secuelas neurológicas a largo plazo y su seguridad cuando se utiliza adecuadamente, debería considerarse su uso rutinariamente.

\section{BIBLIOGRAFÍA}

1. Leskinen K. Complications of acute otitis media in children. Current allergy and asthma reports 2005; 5(4): 308-12. Epub 2005/06/22.

2. Simith JA, Danner CJ. Complications of chronic otitis media and cholesteatoma. Otolaryngologic Clinics of North America 2006; 39(6): 1237-55. Epub 2006/11/14.

3. Manolidis S, KUtzJW, Jr. Diagnosis and management of lateral sinus thrombosis. Otology \& neurotology: official publication of the American Otological Society, American Neurotology Society [and] European Academy of Otology and Neurotology 2005; 26(5): 1045-51. Epub 2005/09/10.

4. Leskinen K, Jero J. Complications of acute otitis media in children in southern Finland. International Journal of Pediatric Otorhinolaryngology 2004; 68(3): 317-24. Epub 2004/05/08.

5. Tov Ee, Leiberman A, Shelef I, Kaplan DM. Conservative nonsurgical treatment of a child with otogenic lateral sinus thrombosis. American Journal of Otolaryngology 2008; 29(2): 138-41. Epub 2008/03/04.

6. Novoa E, Podvinec M, Angst R, Gurtler N. Paediatric otogenic lateral sinus thrombosis: therapeutic management, outcome and thrombophilic evaluation. International Journal of Pediatric Otorhinolaryngology 2013; 77(6): 996-1001. Epub 2013/05/04.

7. Au JK, Adam SI, Michaelides EM. Contemporary management of pediatric lateral sinus thrombosis: a twenty year review. American Journal of
Otolaryngology 2013; 34(2): 145-50. Epub 2012/11/28.

8. Funamura JL, Nguyen at, Díaz RC. Otogenic lateral sinus thrombosis: case series and controversies. International Journal of Pediatric Otorhinolaryngology 2014; 78(5): 866-70. Epub 2014/04/01.

9. KuczKowski J, Dubaniewicz-Wybieralska M, PrzeWozny T, Narozny W, MikaszewskI B. Otitic hydrocephalus associated with lateral sinus thrombosis and acute mastoiditis in children. International Journal of Pediatric Otorhinolaryngology 2006; 70(10): 1817-23. Epub 2006/08/11.

10. Bales CB, Sobol S, Wetmore R, Elden LM. Lateral sinus thrombosis as a complication of otitis media: 10-year experience at the children's hospital of Philadelphia. Pediatrics 2009; 123(2): 709-13. Epub 2009/01/28.

11. Neilan RE, Isaacson B, Kutz JW, JR, Lee KH, Roland PS. Pediatric otogenic lateral sinus thrombosis recanalization. International Journal of Pediatric Otorhinolaryngology 2011; 75(6): 850-3. Epub 2011/04/26.

12. Gutiérrez CC, Carrasco ML, Rahal EM. Trombosis de seno lateral como complicación de otitis media aguda en niños: Reporte de un caso y revisión de la literatura. Revista de Otorrinolaringología y Cirugía de Cabeza y Cuello 2012; 72: 187-94.

13. HiRSCH M, ToRRES GA. Trombosis venosa intracraneal: Signos imagenológicos y errores frecuentes. Revista Chilena de Radiología 2010; 16: 175-87.

14. Bianchini C, Aimoni C, Ceruti S, Grasso DL, Martinı A. Lateral sinus thrombosis as a complication of acute mastoiditis. Acta otorhinolaryngologica Italica: organo ufficiale della Societa italiana di otorinolaringologia e chirurgia cervico-facciale. 2008; 28(1): 30-3. Epub 2008/06/07.

15. Sitton MS, Chun R. Pediatric otogenic lateral sinus thrombosis: role of anticoagulation and surgery. International Journal of Pediatric Otorhinolaryngology 2012; 76(3): 428-32. Epub 2012/01/27. 